\title{
The Notion of Arbitrage and Free Lunch in Mathematical Finance
}

\author{
Walter Schachermayer* \\ Vienna University of Technology and Université Paris Dauphine
}

\begin{abstract}
We shall explain the concepts alluded to in the title in economic as well as in mathematical terms. These notions play a fundamental role in the modern theory of mathematical finance. We start by presenting the ideas in a very informal style and then gradually raise the level of mathematical formalisation.
\end{abstract}

\section{Arbitrage}

The notion of arbitrage is crucial in the modern theory of Finance. It is the cornerstone of the option pricing theory due to F. Black, M. Scholes and R. Merton (published in 1973, Nobel prize in Economics 1997).

The underlying idea is best explained by telling a little joke: A finance professor and a normal person go on a walk and the normal person sees a $€ 100$ bill lying on the street. When the normal person wants to pick it up, the finance professor says: "Don't try to do that! It is absolutely impossible that there is a $€ 100$ bill lying on the street. Indeed, if it were lying on the street, somebody else would already have picked it up before you". (end of joke)

How about financial markets? There it is already much more reasonable to assume that there are no $€ 100$ bills lying around waiting to be picked up. We shall call such opportunities of picking up money that is "lying around" arbitrage possibilities. Let us illustrate this with an easy example.

Consider the trading of $\$$ versus $€$ which takes place simultaneously at two exchanges, say in New York and Paris. Assume for simplicity that in New York the $\$ / €$ rate is $1: 1$. Then it is quite obvious that in Paris the exchange

${ }^{*}$ Support from the Austrian Science Fund (FWF) under Grant P15889 and from Vienna Science and Technology Fund (WWTF) under Grant MA13 is gratefully acknowledged. This is an expanded version of a short note in the Notices of the American Mathematical Society [DS 04]. 
rate (at the same moment of time) also is 1:1. Let us have a closer look why this is indeed the case. Suppose to the contrary that you can buy in Paris a $\$$ for $€ 0,999$. Then, indeed, the so-called "arbitrageurs" (these are people with two telephones in their hands and three screens in front of them) would quickly act to buy $\$$ in Paris and simultaneously sell the same amount of $\$$ in New York, keeping the margin in their (or their bank's) pocket. Note that there is no normalizing factor in front of the exchanged amount and the arbitrageur would try to do this on as large a scale as possible.

It is rather obvious that in the above described situation the market cannot be in equilibrium. A moment's reflection reveals that the market forces triggered by the arbitrageurs acting according to the above scheme will make the $\$$ rise in Paris and fall in New York. The arbitrage possibility will only disappear when the two prices become equal. Of course "equality" here is to be understood as an approximate identity where - even for arbitrageurs with very low (proportional) transaction costs - the above scheme is not profitable any more.

This brings us to a first - still informal and intuitive — definition of arbitrage: an arbitrage opportunity is the possibility to make a profit in a financial market without risk and without net investment of capital. The principle of no arbitrage states that a mathematical model of a financial market should not allow for arbitrage possibilities.

\section{An easy model of a financial market}

To apply this principle to less trivial cases, we consider a - still extremely simple - mathematical model of a financial market: there are two assets, called the bond and the stock. The bond is riskless, hence - by definition - we know what it is worth tomorrow. For (mainly notational) simplicity we neglect interest rates and assume that the price of a bond equals $€ 1$ today as well as tomorrow, i.e.,

$$
B_{0}=B_{1}=1 \text {. }
$$

The more interesting feature of the model is the stock which is risky: we know its value today, say $S_{0}=1$, but we do not know its value tomorrow. We model this uncertainty stochastically by defining $S_{1}$ to be a random variable depending on the random element $\omega \in \Omega$. To keep things as simple as possible, we let $\Omega$ consist of two elements only, $g$ for "good" and $b$ for "bad", with probability $\mathbf{P}[g]=\mathbf{P}[b]=\frac{1}{2}$. We define $S_{1}(\omega)$ to equal 2 or $\frac{1}{2}$ according to whether

$$
S_{1}(\omega)= \begin{cases}2 & \text { for } \omega=g \\ \frac{1}{2} & \text { for } \omega=b\end{cases}
$$

Now we introduce a third financial instrument in our model, an option on the stock with strike price $K$ : the buyer of the option has the right — but not 
the obligation - to buy one stock at time $t=1$ at the predefined price $K$. To fix ideas let $K=1$. A moment's reflexion reveals that the price $C_{1}$ of the option at time $t=1$ (where $C$ stands for contingent claim) equals

$$
C_{1}=\left(S_{1}-K\right)_{+},
$$

i.e., in our simple example

$$
C_{1}(\omega)= \begin{cases}1 & \text { for } \omega=g \\ 0 & \text { for } \omega=b\end{cases}
$$

Hence we know the value of the option at time $t=1$, contingent on the value of the stock. But what is the price of the option today?

At this stage the reader might consult the financial section of a newspaper or the web to see some "life" examples on quoted option prices.

The classical approach, used by actuaries for centuries, is to price contingent claims by taking expectations, which leads to the value $C_{0}:=\mathbf{E}\left[C_{1}\right]=\frac{1}{2}$ in our example. Although this simple approach is very successful in many actuarial applications, it is not at all satisfactory in the present context. Indeed, the rationale behind taking the expected value as the price of a contingent claim is the following: in the long run the buyer of an option will neither gain nor lose on average. We rephrase this fact in a financial lingo: the performance of an investment in the option would on average equal the performance of the bond. However, a basic feature of finance is that an investment into a risky asset should, on average, yield a better performance than an investment in the bond (for the skeptical reader: at the least these two values should not necessarily coincide). In our "toy example" we have chosen the numbers such that $\mathbf{E}\left[S_{1}\right]=1.25>1=\mathbf{E}\left[B_{1}\right]$, so that on average the stock performs better than the bond.

\section{Pricing by No Arbitrage}

A different approach to the pricing of the option goes like this: we can buy at time $t=0$ a portfolio consisting of $\frac{2}{3}$ of stock and $-\frac{1}{3}$ of bond. The reader might be puzzled about the negative sign: investing a negative amount in a bond - "going short" in financial lingo - means to borrow money.

One verifies that the value $\Pi_{1}$ of the portfolio at time $t=1$ equals 1 or 0 depending on whether $\omega$ equals $g$ or $b$. The portfolio "replicates" the option, i.e.,

$$
C_{1} \equiv \Pi_{1} .
$$

We are confident that the reader now sees why we have chosen the above weights $\frac{2}{3}$ and $-\frac{1}{3}$ : the mathematical complexity of determining these weights such that (5) holds true amounts to solving two linear equations in two variables. 
The portfolio $\Pi$ has a well-defined price at time $t=0$, namely $\Pi_{0}=$ $\frac{2}{3} S_{0}-\frac{1}{3} B_{0}=\frac{1}{3}$. Now comes the "pricing by no arbitrage" argument: equality (5) implies that we also must have

$$
C_{0}=\Pi_{0}
$$

whence $C_{0}=\frac{1}{3}$. Indeed, suppose that (6) does not hold true; to fix ideas, suppose we have $C_{0}=\frac{1}{2}$ as above. This would allow an arbitrage by buying ("going long in") the portfolio $\Pi$ and simultaneously selling ("going short in") the option $C$. The difference $C_{0}-\Pi_{0}=\frac{1}{6}$ remains as arbitrage profit at time $t=0$, while at time $t=1$ the two positions cancel out independently of whether the random element $\omega$ equals $g$ or $b$.

\section{Variations of the example}

Although the preceding "toy example" is extremely simple and, of course, far from reality, it contains the heart of the matter: the possibility of replicating a contingent claim, e.g. an option, by trading on the existing assets and applying the no arbitrage principle.

It is straightforward to generalize the example by passing from the time index set $\{0,1\}$ to an arbitrary finite discrete time set $\{0, \ldots, T\}$ by considering $T$ independent Bernoulli random variables. This binomial model is called the Cox-Ross-Rubinstein model in finance. It is not difficult - at least with the technology of stochastic calculus that is available today - to pass to the (properly normalized) limit as $T$ tends to infinity, thus ending up with a stochastic process driven by Brownian motion. The so-called geometric Brownian motion with drift is the celebrated Black-Scholes model, which was proposed in 1965 by P. Samuelson. In fact, already in 1900 L. Bachelier used Brownian motion to prize options in his remarkable thesis "Théorie de la spéculation" [B 00] (member of the jury and rapporteur: H. Poincaré).

In order to apply the above no arbitrage arguments to more complex models we still need one more crucial concept.

\section{$5 \quad$ Martingale Measures}

To explain this notion let us turn back to our "toy example", where we have seen that the unique arbitrage-free price of our option equals $C_{0}=\frac{1}{3}$. We also have seen that, by taking expectations, we obtained $\mathbf{E}\left[C_{1}\right]=\frac{1}{2}$ as the price of the option, which allowed for arbitrage possibilities. The economic rationale for this discrepancy was that the expected return of the stock was higher than that of the bond.

Now make the following thought experiment: suppose that the world is governed by a different probability than $\mathbf{P}$ that assigns different weights to $g$ 
and $b$, such that under this new probability - let's call it $\mathbf{Q}$ - the expected return of the stock equals that of the bond. An elementary calculation reveals that the probability measure defined by $\mathbf{Q}[g]=\frac{1}{3}$ and $\mathbf{Q}[b]=\frac{2}{3}$ is the unique solution satisfying $\mathbf{E}_{\mathbf{Q}}\left[S_{1}\right]=S_{0}=1$. Speaking mathematically, the process $S$ is a martingale under $\mathbf{Q}$, and $\mathbf{Q}$ a martingale measure for $S$.

Speaking again economically, it is not unreasonable to expect that in a world governed by $\mathbf{Q}$, the recipe of taking expected values should indeed give a price for the option that is compatible with the no arbitrage principle. A direct calculation reveals that in our "toy example" this is indeed the case:

$$
\mathbf{E}_{\mathbf{Q}}\left[C_{1}\right]=\frac{1}{3}
$$

At this stage it is, of course, the reflex of every mathematician to ask: what precisely is going on behind this phenomenon?

\section{The Fundamental Theorem of Asset Pricing}

The basic message of this theorem is that - essentially — a model of a financial market is free of arbitrage if and only if there is a probability measure $\mathbf{Q}$, equivalent to the original $\mathbf{P}$ (i.e., $\mathbf{P}[A]=0$ iff $\mathbf{Q}[A]=0$ ), such that the stock price process is a martingale under $\mathbf{Q}$. In this case the recipe of taking expectations $\mathbf{E}_{\mathbf{Q}}[$.$] in order to price contingent claims yields precisely the$ arbitrage-free pricing rules, where $\mathbf{Q}$ runs through all equivalent martingale measures. In particular, if $\mathbf{Q}$ is unique, $\mathbf{E}_{\mathbf{Q}}[$.] yields the unique arbitrage-free price, as in the "toy example" above.

This theorem was proved by M. Harrison and S. Pliska [HP 81] in 1981 for the case where the underlying probability space $(\Omega, \mathcal{F}, \mathbf{P})$ is finite. In the same year D. Kreps [K 81] extended this theorem to a more general setting: for this extension the condition of no arbitrage turns out to be too narrow and has to be replaced by a stronger assumption.

\section{$7 \quad$ No Free Lunch}

We formalize the model of a financial market in continuous time $[0, T]$ : the bond again is harmless and without loss of generality normalized, i.e., $B_{t} \equiv 1$ for $0 \leq t \leq T$. The stock price process $\left(S_{t}\right)_{0 \leq t \leq T}$ is assumed to be an $\mathbb{R}^{d_{-}}$ valued stochastic process (we consider $d=1$ for simplicity) defined over and adapted to a filtered stochastic base $\left(\Omega, \mathcal{F},\left(\mathcal{F}_{t}\right)_{0 \leq t \leq T}, \mathbf{P}\right)$ such that the filtration $\left(\mathcal{F}_{t}\right)_{0 \leq t \leq T}$ satisfies the "usual conditions" of right continuity and saturatedness.

A real-valued stochastic process $S=\left(S_{t}\right)_{0 \leq t \leq T}$ is a function $S: \Omega \times[0, T] \rightarrow$ $\mathbb{R}$ verifying some measurability condition: we call $S$ progressively measurable 
if, for every $t \in[0, T]$, the restriction of $S$ to $\Omega \times[0, t]$ is $\mathcal{F}_{t} \otimes \operatorname{Borel}([0, t])$ measurable. The interpretation is that the behaviour of $\left(S_{u}\right)_{0 \leq u \leq t}$ depends only on the information available at time $t$, which is modelled by the sigma-algebra $\mathcal{F}_{t}$.

The "economic agents" are allowed to buy or sell arbitrary quantities of the stock during the time interval $[0, T]$. To model this activity mathematically, first consider so-called "elementary" trading strategies: fix a mesh $0=t_{0}<$ $t_{1}<\ldots<t_{n}=T$, which we interpret as the (deterministic) instants of time when the agent rebalances her portfolio. To define the portfolio she has to determine the amounts $H_{t_{i-1}}$ of stock which she holds during the time intervals ]$\left.t_{i-1}, t_{i}\right]$. When she will make this decision, i.e. at time $t_{i-1}$, she will dispose of the information available at at time $t-1$; hence it is natural to require $H_{t_{i-1}}$ to be an $\mathcal{F}_{t_{i-1}}$-measurable random variable. An elementary trading strategy therefore is defined as a function $H=H_{t}(\omega)$ defined on $\Omega \times T$ of the form

$$
H_{t}(\omega)=\sum_{i=1}^{n} H_{t_{i-1}}(\omega) \mathbf{1}_{\left[t_{i-1}, t_{i}\right.}
$$

where each $H_{t_{i-1}}$ is $\mathcal{F}_{t_{i-1}}$-measurable. The interpretation is that at time $t$ the agent holds $H_{t}(\omega)$ units of the stock in her portfolio.

For each such $H$ we may define the stochastic integral $X_{T}^{H}$ as the random variable

$$
X_{T}^{H}=\sum_{i=1}^{n} H_{t_{i-1}}\left(S_{t_{i}}-S_{t_{i-1}}\right)=: \int_{0}^{T} H_{t} d S_{t}
$$

The interpretation is as follows: applying the trading strategy $H$ results in a (random) gain or loss $H_{t_{i-1}}(\omega)\left(S_{t_{i}}(\omega)-S_{t_{i-1}}(\omega)\right)$ during the time interval ]$\left.t_{i-1}, t_{i}\right]$. The total gain or loss therefore is given by the above Riemann-type sum which may formally be written — for each fixed $\omega \in \Omega$ - as a Stieltjes integral $d S_{t}$ over a step function.

This notation insinuates already that one should allow for more general trading strategies than just "elementary" ones. Similarly as in ordinary calculus the step functions are only a technical gimmick on the way to a reasonable integration theory.

In order to pass to more general integrands $H$ in (9) we need some more steps: firstly we have to suppose that $S$ is a semi-martingale (see [RY 91] for a definition). This is, according to a theorem of Bichteler and Dellacherie, the maximal class of stochastic processes for which there is a reasonable integration theory. Fortunately, this assumption of $S$ being a semi-martingale does not restrict the generality: indeed it was shown in [DS 94] that, whenever $S$ fails to be a semi-martingale then - essentially (in a sense made precise in [DS 94]) - $S$ admits arbitrage.

We can now proceed developping the integration theory. It is natural to replace the deterministic mesh $0=t_{0} \leq \ldots \leq t_{n}=T$ by an increasing 
sequence of stopping times $0=\tau_{0} \leq \ldots \leq \tau_{n}=T$. The corresponding class $\mathcal{H}$ of integrands (or trading strategies in the financial lingo) is called the class of simple integrands.

This first extension step (from elementary to simple integrands) is rather harmless and does not require any delicate passage to the limit as the integral is still defined by a finite sum as in (9).

In general, trading strategies are modeled by predictable, $S$-integrable processes $\left(H_{t}\right)_{0 \leq t \leq T}$, where $H_{t}$ describes the amount of the stock held at time $t$. For each $H$ the random variable

$$
X_{T}^{H}=\int_{0}^{T} H_{t} d S_{t}
$$

then equals the accumulated gains or losses up to time $T$ by following the trading strategy $H$. For technical reasons we have to restrict ourselves to "admissible" trading strategies $H$ : we require that there is some constant $M$ (a "credit line") such that, for all $0 \leq t \leq T$, the accumulated losses up to time $t$ are less than $M$ almost surely,

$$
\int_{0}^{t} H_{u} d S_{u}>-M, \quad \text { a.s., for } 0 \leq t \leq T \text {. }
$$

We now may formally define an arbitrage opportunity as an admissible trading strategy $H$ s.t. the random variable $X_{T}^{H}$ is non-negative a.s. and strictly positive with strictly positive probability.

We have mentioned above that the requirement that there are no arbitrage possibilities is too weak to imply the existence of an equivalent martingale measure in general. Kreps' idea was to allow for a passage to the limit:

Definition 7.1 [K 81] The process $S$ admits a free lunch, if there is a random variable $f \in L_{+}^{\infty}(\Omega, \mathcal{F}, \mathbf{P})$ with $\mathbf{P}[f>0]>0$ and a net $\left(f_{\alpha}\right)_{\alpha \in I}=\left(g_{\alpha}-h_{\alpha}\right)_{\alpha \in I}$ such $g_{\alpha}=\int_{0}^{T} H_{t}^{\alpha} d S_{t}$, for some admissible trading strategy $H^{\alpha}, h_{\alpha} \geq 0$ and $\left(f_{\alpha}\right)_{\alpha \in I}$ converges to $f$ in the weak-star topology of $L^{\infty}(\Omega, \mathcal{F}, \mathbf{P})$.

The economic idea behind this notion is the following: although $f$ itself is not supposed to be of the form $\int_{0}^{T} H_{t} d S_{t}$, for some admissible $H$ (this would be an arbitrage), we require that $f$ can be approximated by $f_{\alpha}$ in a suitable topology. The interpretation of the random variables $h_{\alpha} \geq 0$ is that, in this approximation, people are allowed to "throw money away".

With these preparations we can deduce from D. Kreps' work the following version of the fundamental theorem of asset pricing.

Theorem 7.2 A bounded process $S=\left(S_{t}\right)_{0 \leq t \leq T}$ admits no free lunch iff there is a probability measure $\mathbf{Q}$ equivalent to $\mathbf{P}$ such that $S$ is a martingale under Q. 
Subsequent to Kreps' seminal paper many authors elaborated on improvements of this theorem (see, e.g., [DS 98] for an account on the literature). Typical questions are whether the weak-star topology (which is difficult to interpret economically) can be replaced by a finer topology or whether the $\operatorname{net}\left(f_{\alpha}\right)_{\alpha \in I}$ can be replaced by a sequence $\left(f_{n}\right)_{n \in \mathbb{N}}$.

In [DS 94] we introduced the notion of a "free lunch with vanishing risk" by replacing the weak-star topology in the above definition by the norm-topology of $L^{\infty}$. One may then replace the net $\left(f_{\alpha}\right)_{\alpha \in I}$ by a sequence $\left(f_{n}\right)_{n=1}^{\infty}$; this notion allows for a clear-cut economic interpretation to which we tried to allude to by the term "vanishing risk".

To extend to the case of unbounded processes (which are important in the applications) one also needs some generalisations of the notions of a martingale, namely the notion of local martingales and sigma-martingales. The latter concept goes back to the work of Chou and Emery (see [DS 98]).

We now can state the version of the fundamental theorem of asset pricing as obtained in [DS 98].

Theorem 7.3 A semi-martingale $S=\left(S_{t}\right)_{0 \leq t \leq T}$ admits no free lunch with vanishing risk iff there is a probability measure $\mathbf{Q}$ equivalent to $\mathbf{P}$ such that $S$ is a sigma-martingale under $\mathbf{Q}$.

If $S$ is bounded (resp. locally bounded) the term sigma-martingale may equivalently be replaced by the term martingale (resp. local martingale).

\section{References}

[B 00] L. Bachelier, (1900), Théorie de la Speculation. Ann. Sci. Ecole Norm. Sup., Vol. 17, pp. 21-86, [English translation in: The Random Character of stock prices (P. Cotner, editor), MIT Press, 1964].

[BS 73] F. Black, M. Scholes, (1973), The pricing of options and corporate liabilities. Journal of Political Economy, Vol. 81, pp. 637-659.

[HP 81] J.M Harrison, S.R. Pliska, (1981), Martingales and Stochastic intefrals in the theory of continuous trading. Stoch. Proc. \& Appl., Vol. 11, pp. 215-260.

[K 81] D.M. Kreps, (1981), Arbitrage and equilibrium in economies with infinitely many commodities. J. Math. Econ., Vol. 8, pp. 15-35.

[DS 94] F. Delbaen, W. Schachermayer, (1994), A General Version of the Fundamental Theorem of Asset Pricing. Math. Annalen, Vol. 300, pp. $463-520$. 
[DS 98] F. Delbaen, W. Schachermayer, (1998), The Fundamental Theorem of Asset Pricing for Unbounded Stochastic Processes. Mathematische Annalen, Vol. 312, pp. 215-250.

[DS 04] F. Delbaen, W. Schachermayer, (2004), What is a Free Lunch? Notices of the AMS, Vol. 51, No. 5, pp. 526-528.

[RY 91] D. Revuz, M. Yor, (1991), Continuous Martingales and Brownian Motion. Springer, Berlin Heidelberg New York. 